\title{
Article \\ Doctors and Nurses: A Systematic Review of the Risk and Protective Factors in Workplace Violence and Burnout
}

\author{
Jose Miguel Giménez Lozano ${ }^{1}$, Juan Pedro Martínez Ramón ${ }^{2} \mathbb{D}$ and Francisco Manuel Morales Rodríguez ${ }^{1, *}$ \\ 1 Department of Educational and Developmental Psychology, Faculty of Psychology, \\ Campus Universitario de Cartuja, University of Granada, 18071 Granada, Spain; josemi1992@correo.ugr.es \\ 2 Department of Developmental and Educational Psychology, Campus of Espinardo, University of Murcia, \\ 30100 Murcia, Spain; juanpedromartinezramon@um.es \\ * Correspondence: fmmorales@ugr.es; Tel.: +34-68-097-6924
}

\section{check for} updates

Citation: Giménez Lozano, J.M.; Martínez Ramón, J.P.; Morales Rodríguez, F.M. Doctors and Nurses: A Systematic Review of the Risk and Protective Factors in Workplace Violence and Burnout. Int. J. Environ. Res. Public Health 2021, 18, 3280. https://doi.org/10.3390/ ijerph18063280

Academic Editors: Paul Tchounwou and María del Carmen Pérez-Fuentes

Received: 31 December 2020

Accepted: 15 March 2021

Published: 22 March 2021

Publisher's Note: MDPI stays neutral with regard to jurisdictional claims in published maps and institutional affiliations.

Copyright: (c) 2021 by the authors. Licensee MDPI, Basel, Switzerland. This article is an open access article distributed under the terms and conditions of the Creative Commons Attribution (CC BY) license (https:/ / creativecommons.org/licenses/by/ $4.0 /)$.

\begin{abstract}
The present study aims analyze the risk factors that lead to high levels of burnout among nurses and physicians and the protective factors that prevent them. Thus, it is also intended to explore the possible correlation between physical and verbal violence produced at work and the symptoms derived from burnout. Methods: The search was carried out on the Scopus, PubMed and Web of Science databases between 2000 and 2019 (on which date the bibliographic search ends). Descriptive studies estimating the prevalence of workplace violence and risk and protective factors and burnout were included. An adapted version of the Downs and Black quality checklist was used for article selection. 89.6 percent of the studies analysed were in the health sector. There is a significant correlation between burnout symptoms and physical violence at work. On the one hand, the risk factors that moderate this correlation were of structural/organisational type (social support, quality of the working environment, authoritarian leadership, little autonomy or long working days, etc.) and personal type (age, gender, nationality or academic degree, etc.). On the other hand, protective factors were the quality of the working environment, mutual support networks or coping strategies. The results were analysed in-depth and intervention strategies were proposed.
\end{abstract}

Keywords: burnout; workplace violence; protective factors; risk factors

\section{Introduction}

Workplace violence (WV) is understood as any type of act, incident, or behavior in which a person is abused, threatened, humiliated, or assaulted in the workplace, including verbal and physical assaults [1]. The National Institute of Safety and Hygiene in the Workplace (Spanish acronym: INSHT) affirms that WV is one of the main health risk factors for those who are active in the workplace, since such actions result in a multitude of psychological and emotional conditions that prevent the development of a 'working life or aggravate the problems that occur regardless of work. The many studies related to $\mathrm{WV}$ have observed that the risks of exposure to this type of situation are very high in those jobs that imply providing a service to people, so the nursing and medical profession would stand out for the nature of one's own work as a facilitator for the creation of WV situations [2]. The WV is characterized by being diverse in its ways of expressing itself, physically (hitting, pushing, kicking ... ) verbally (yelling, insults, offensive comments in general ... ) or sexually [3].

Previous models related to working conditions and the work environment assumed that workplace violence was based exclusively on situations involving physical violence, leaving aside stress, overwork, or the psychological demands of the task itself [4]. Since then, many authors have focused their work on discovering the causes and consequences that it occurs 'daily'. For example, Mucci [5] observed that working conditions such as high work demands, job control, type of leadership, peer support, company organization, and even gender (higher among women than among men) correlated with WV. Following 
the same line of research, but focusing on individual factors, Robelski [6] showed that emotional demands, job satisfaction, and each person's own resilience had a direct influence on WV. On the other hand, the consequences generated by violence at work are related to the high levels of stress experienced. Thus, the vast majority of studies have found that workers who have repeatedly suffered some type of aggression at work present high levels of anxiety, depression, generalized fear, insomnia or emotional problems that lead to more serious disorders such as Post-Traumatic Stress Disorder or burnout [7].

The concept of burnout has been closely related to the adverse effects produced by the labor sector since 1970 [8], and is usually defined as "a frequent physical and emotional exhaustion of workers, especially those who provide some type of service to others, resulting from the conditions and risk situations experienced." Maslach and Jackson were the first to define burnout. They developed an instrument to assess the burnout that occurred in three areas using the Maslach Burnout Inventory (MBI) [9] and which is composed of three dimensions: (1) Emotional Exhaustion (EE) related to mental and emotional fatigue, (2) Depersonalization, related to the most negative behaviors of individuals, (3) and low Personal Accomplishment (PA), meaning a tendency to be evaluated negatively based on job performance [10]. Subsequently, in 1992, the International Statistical Classification of Diseases and Related Health Problems define the burnout under the heading "Problems related to life management difficulty" (Z73) and is defined as a "State of vital exhaustion" (ICD-10, 2012) [11]. The American Psychiatric Association (APA) does not yet include a definition of burnout in its Diagnostic and Statistical Manual-V (DSM-V, 2013) [12]. This disorder is often characteristic of the most stressful jobs, which involve constant direct contact with other people such as police officers, teachers and especially health sector employees such as nurses, doctors, assistants, etc. [12]. Burnout has consequences on the physical and mental health of nurses, workplace; for example, this may affect the individual, generating physical symptoms of fatigue, anxiety, sleep disorders, insomnia, headaches, and frequent colds alongside reduced concentration and memory [13], also affecting work levels, such as absenteeism or intention to leave [14].

Nurses have been considered a risk group because of the high levels of burnout they suffer daily [15]. For example, it has been observed that nurses would have a much higher prevalence of burnout than other sectors [16] due to frequent understaffing in hospitals that increases the nurse-to-patient ratio, high work overload, long working days and long working hours that vary from day to day [17]. In addition, it has been observed that health sector personnel, especially those working in higher intensity facilities such as those involving emergencies, palliative care, or ambulance services, are noted for a high percentage of aggressions either by a patient or a colleague [18]. These assaults can be verbal or physical, although sexual assaults on female health workers are also very common [19].

On the other hand, doctors are another group to consider due to the high prevalence shown by the results of several investigations. A systematic review by Rotenstein, Torre, Ramos [20] of 182 articles showed a prevalence of $0 \%$ to $86.2 \%$ in mental exhaustion among physicians, $0 \%$ to $89.9 \%$ in depersonalization, and $0 \%$ to $87.1 \%$ in low personal accomplishment.

The aim of this work is to analyze the risk factors that lead to high levels of burnout levels among nurses and physicians, and the protective factors that can help prevent burnout.

\section{Materials and Methods}

A bibliographic review has been carried out following PRISMA's recommendations for descriptive and systematic reviews.

\subsection{Search Strategies}

During the second and third quarters of 2020, an exhaustive search was carried out in the databases of Scopus, Pubmed, WOS, PsycINFO and Cochrane. A free search was carried out in Google Scholar and in portals related to occupational health and healthcare 
at work to identify those studies that were not published in the databases (13.2\%). The search strategy was based on the combination of specific search terms: Violence [Mesh], Workplace Violence [Mesh], Health Personnel [Mesh], Burnout, protective factors, risk factors, medical staff.

\subsection{Data Extraction}

Data extraction was performed using a standard data extraction form developed by the Joanna Briggs Institute Reviewers' Manual for The Systematic Review of Prevalence and Incidence Data [21]. We used a specific coding manual to extract information from the primary studies. To ensure rigor in the final selection of studies, the three reviewers ran the form through all of the articles to check their suitability, relevance and precision.

\subsection{Inclusion Criteria}

Studies were included according to the following criteria: (a) empirical; (b) published between 2000 and 2019 (on which date the bibliographic search ends); (c) empirical studies; (d) published in Spanish, Catalan or English; (e) the articles must evaluate t burnout syndrome with the standardized Maslach Burnout Inventory questionnaire [22]; (f) compare burnout levels in medical and nursing professionals; (g) evaluating at least one type of WV (physical, verbal or sexual); (h) aiming to observe the protective and risk factors related to WV. Articles of an informative nature, experts' opinions published in editorials and letters to the editor were excluded (Figure 1). The diagram was made following the PRISMA declaration [23].

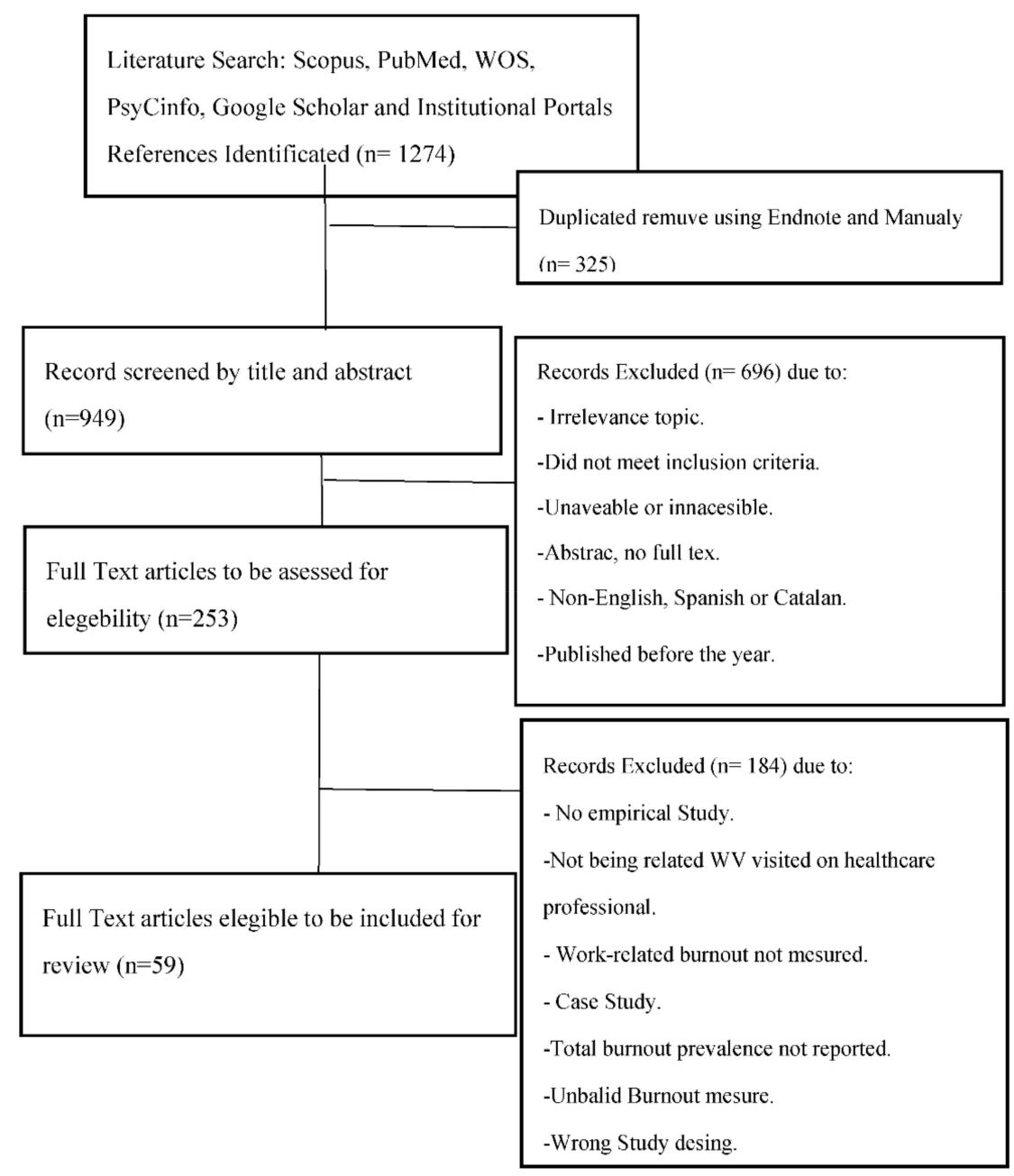

Figure 1. PRISMA flow Chart. 


\subsection{Selection of the Studies}

The main search yielded a result of 949 posts. The first selection phase was carried out based on reading the titles and abstracts of the publications; 253 articles were selected for further study. The exclusion criteria in this first phase were: non-empirical studies, not published before the year 2000, not written in Spanish, Catalan or English, not being directly related to workplace violence towards health professionals and not evaluating burnout with the MBI. The selection was then examined by means of a complete reading of the articles taking into account the inclusion criteria. Finally, 59 documents were selected for their final review. $13.2 \%$ of the final selection came from Google Scholar and institutional portals.

\subsection{Data Analysis Section}

Variables and information extracted from the studies. Literature search results and data extraction results were summarized descriptively. To exclude duplicate articles, we used the program Endnote for manual selection. A summary of efficacy outcomes was presented based on the different outcome measures, controls and interventions. A narrative synthesis was therefore generated, considering the total number of studies that reported results, the methodological quality, and the quality of evidence for the outcomes to yield final conclusions. The general characteristics of systematic reviews extracted were used as exploratory variable PRISMA scores. We included: number of authors, nurses versus physicians, percentage of women versus men, transversal studies versus other methodological interventions, type of burnout and WV instrumentals used in the study, presence of risk and protectives factors versus absence. If the value of these factors was $p$-value $<0.05$, it was considered statistically significant for the systematic review.

\section{Results}

The results of the final selection of the studies are show on Table 1 (See Table 1). The information in Table 1 allowed us to calculate the statistics. The descriptive results are shown on Table 2 (See Table 2). The total population analysed was 22,993 $(N=22,993)$, with a mean age of $34.18($ Mean $=34.18 ; S D=6.14)$. A higher women's employment rate can be observed in the world health sector, representing $61.43 \%(N=14,126)$ of all studies, men being at $38.56 \%(N=8867)$. Most of the research related to burnout and WV is focused on the nursing profession, with $71.64 \%(N=16,473)$ representing the nurses who have been evaluated. Doctors appear to take up $28.49 \%(N=6551)$ of the research, and a lower percentage is taken up by nursing assistants, orderlies and/or technicians, $1.16 \%(N=269)$. The most evaluated specialties are the ICU or Intensive Care Unit (Critical Care) $(N=9344$; $\%=40.63 \%)$, Emergencies $(N=6719 ; \%=29.22 \%)$, the Mental Health specialty $(N=4236$; $\%=18.54 \%)$ and Surgery $(N=4187 ; \%=18.45 \%)$. In terms of work contracts, those of a fixed or full-time nature are the most frequent, $57.66 \%(N=13,258)$, as are, to a lesser extent, part-time $-28.07 \%(N=6456)$-and temporary contracts-14.02 $(N=3279)$. The university academic level is most common among the population examined, $38.02 \%(N=8793)$, as is experience of between 5 and 15 years, $42.11 \%(N=9683)$. 
Table 1. Description of the included studies.

\begin{tabular}{|c|c|c|c|c|c|c|c|c|}
\hline Autores & $n$ & Age & $\%$ Women & Type Work & Type Study & Burnout Evaluation & $\begin{array}{l}\text { Workplace Violence } \\
\text { Evaluation }\end{array}$ & Otras Medidas \\
\hline (Out, 2005) [24] & 385 & 41 & $97.2 \%$ & Nurses & Transversal & $\begin{array}{l}\text {-Maslach Burnout } \\
\text { Inventory-Human } \\
\text { Services Survey } \\
\text { (MBI-HSS) }\end{array}$ & $\begin{array}{l}\text {-The Negative Acts } \\
\text { Questionnaire (NAQ). } \\
\text {-Specific Events in the } \\
\text { Workplace (Keashly, Trott, \& } \\
\text { MacLean, 1994). }\end{array}$ & $\begin{array}{l}\text { Job Satisfaction Subscale of the Ward } \\
\text { Organisational Features Scales (WOFS). } \\
\text {-Turnover Cognitions Scale (Bozeman \& } \\
\text { Perrewe, 2001). } \\
\text {-World Assumptions Scale (WAS; Janoff-Bulman, } \\
\text { 1989): Evalúa los factores relacionados con la } \\
\text { benevolencia. } \\
\text {-Symptom Assessment-45 Questionnaire } \\
\text { (SA-4): Evalúa aspectos psicológicos. }\end{array}$ \\
\hline (Merezc, 2006) [25] & 413 & 38 & $x$ & Physicians & Transversal & $\begin{array}{l}\text {-Maslach Burnout } \\
\text { Inventory (MBI) }\end{array}$ & $\begin{array}{l}\text { Own questionnaire on } \\
\text { assaults suffered at work. }\end{array}$ & -General Health Questionnaire (GHQ-28) \\
\hline $\begin{array}{c}\text { (Isaksson, 2008) } \\
{[26]}\end{array}$ & 196 & 41.5 & $85.7 \%$ & Assistants & Transversal & $\begin{array}{l}\text {-Maslach Burnout } \\
\text { Inventory-Human } \\
\text { Services Survey } \\
\text { (MBI-HSS) }\end{array}$ & $\begin{array}{l}\text { Own questionnaire on } \\
\text { assaults suffered at work. }\end{array}$ & $\begin{array}{l}\text {-Memories of Parental Rearing } \\
\text {-Temperament and Character Inventory } \\
\text { (TCI-125) }\end{array}$ \\
\hline $\begin{array}{c}\text { (Merecz, 2009) } \\
{[27]}\end{array}$ & 1554 & 39.54 & $x$ & Nurses & Transversal & $\begin{array}{l}\text {-Maslach Burnout } \\
\text { Inventory (MBI) }\end{array}$ & $\begin{array}{l}\text {-Workplace Aggression } \\
\text { Questionnaire (EWAQ). } \\
\text {-Direct reactions to aggressive } \\
\text { behaviours (DRAB). }\end{array}$ & $\begin{array}{l}\text {-Job satisfaction: 22-itemdeveloped by the Work } \\
\text { Psychology Department of NIOM. } \\
\text {-Well-being psychological: General Health } \\
\text { Questionnaire (GHQ-28). }\end{array}$ \\
\hline $\begin{array}{l}\text { (Spence-Laschinger, } \\
2010) \\
{[28]}\end{array}$ & 415 & 27.24 & $94 \%$ & Nurses & Transversal & $\begin{array}{l}\text {-Maslach Burnout } \\
\text { Inventory (MBI) }\end{array}$ & $\begin{array}{l}\text {-Negative Acts } \\
\text { Questionnaire-Revised } \\
\text { (NAQ-R) }\end{array}$ & $\begin{array}{l}\text {-The Authentic Leadership Questionnaire (ALQ) } \\
\text { (Avolio, Gardner, \& Walumbwa, 2007). } \\
\text {-Psychological Capital Questionnaire (Luthans } \\
\text { et al., 2007) }\end{array}$ \\
\hline $\begin{array}{c}\text { (Dikmetas, 2011) } \\
\text { [29] }\end{array}$ & 270 & 30 & $34.07 \%$ & Physicians & Transversal & $\begin{array}{l}\text {-Maslach Burnout } \\
\text { Inventory (MBI) }\end{array}$ & $\begin{array}{l}\text {-Leymann Inventory of } \\
\text { Psychological Terror: Evalúa } \\
\text { el bullying y el mobbing }\end{array}$ & Demographic and professional background. \\
\hline $\begin{array}{c}\text { (Happell, 2011) } \\
{[30]}\end{array}$ & 123 & 41.38 & $x$ & Nurses & Transversal & $\begin{array}{l}\text {-Maslach Burnout } \\
\text { Inventory (MBI) }\end{array}$ & $\begin{array}{l}\text {-Minnesota Satisfaction } \\
\text { Questionnaire (MSQ). }\end{array}$ & $\begin{array}{l}\text {-Survey of Nurses' Attitudes to Seclusion } \\
\text { Survey (SNASS; Heyman 1987). } \\
\text {-Elsom Therapeutic Optimism Scale (ETOS; } \\
\text { Elsom \& McCauley-Elsom 2008) }\end{array}$ \\
\hline $\begin{array}{c}\text { (Murillo, 2011) } \\
\text { [31] }\end{array}$ & 20 & 31 & $45 \%$ & Physicians & Transversal & $\begin{array}{l}\text {-Maslach Burnout } \\
\text { Inventory (MBI-HSS) }\end{array}$ & $\begin{array}{l}\text {-Protocol of assaults on } \\
\text { doctors that evaluates data } \\
\text { on the assault }\end{array}$ & Demographic and professional background. \\
\hline
\end{tabular}


Table 1. Cont

\begin{tabular}{|c|c|c|c|c|c|c|c|c|}
\hline Autores & $n$ & Age & $\%$ Women & Type Work & Type Study & Burnout Evaluation & $\begin{array}{l}\text { Workplace Violence } \\
\text { Evaluation }\end{array}$ & Otras Medidas \\
\hline $\begin{array}{c}\text { (Spence Laschinger, } \\
\text { 2011) } \\
{[32]}\end{array}$ & 165 & 28.28 & $93.2 \%$ & Nurses & Transversal & $\begin{array}{l}\text {-Maslach Burnout } \\
\text { Inventory (MBI) }\end{array}$ & $\begin{array}{l}\text { Negative Acts Ques- } \\
\text { tionnaire-Revised (NAQ-R) }\end{array}$ & $\begin{array}{l}\text {-The Areas of Worklife Scale (AWS). } \\
\text {-Psychological Capital Questionnaire (PCQ). } \\
\text {-Pressure Management Indicator(PMI) }\end{array}$ \\
\hline $\begin{array}{c}\text { (Patrick, 2011) } \\
{[33]}\end{array}$ & 339 & 28.10 & $91.5 \%$ & Nurses & Transversal & $\begin{array}{l}\text {-Maslach Burnout } \\
\text { Inventory (MBI) }\end{array}$ & $\begin{array}{l}\text { Negative Acts Ques- } \\
\text { tionnaire-Revised (NAQ-R) }\end{array}$ & $\begin{array}{l}\text {-The Authentic Leadership Ques-tionnaire } \\
\text { (ALQ) } \\
\text {-Job Satisfaction scale. }\end{array}$ \\
\hline (Sundin, 2011) & 1216 & 42.37 & $94.3 \%$ & Nurses & Longitudinal & $\begin{array}{l}\text {-Maslach Burnout } \\
\text { Inventory (MBI) }\end{array}$ & $\begin{array}{l}\text {-Work Environment Survey } \\
\text { (SWES) }\end{array}$ & $\begin{array}{l}\text {-Work and social support: Items extracted from } \\
\text { the Swedish Work Environment Survey (SWES). }\end{array}$ \\
\hline $\begin{array}{c}\text { (Hensel, 2012) } \\
{[35]}\end{array}$ & 926 & 39.7 & $82.3 \%$ & $\begin{array}{l}\text { Nurses, } \\
\text { physicians and } \\
\text { assistants }\end{array}$ & Transversal & $\begin{array}{l}\text {-Maslach Burnout } \\
\text { Inventory-Human } \\
\text { Services Survey } \\
\text { (MBI-HSS) }\end{array}$ & $\begin{array}{l}\text {-Questionnaire on the } \\
\text { frequency of assaults } \\
\text { suffered at work. }\end{array}$ & -Demographic and professional background. \\
\hline $\begin{array}{c}\text { (Gascon, 2012) } \\
{[36]}\end{array}$ & 1826 & 41.84 & $64.2 \%$ & $\begin{array}{l}\text { Nurses, } \\
\text { physicians and } \\
\text { assistants }\end{array}$ & Transversal & $\begin{array}{l}\text { Maslach Burnout } \\
\text { Inventory (MBI-HSS) }\end{array}$ & $\begin{array}{l}\text { Workplace violence } \\
\text { Questionnaire (Gascón et al. } \\
\text { 2009b) }\end{array}$ & $\begin{array}{l}\text {-Areas of Work-life Scale (AWS, Leiter \& } \\
\text { Maslach 2004a) }\end{array}$ \\
\hline $\begin{array}{c}\text { (Pranjic, 2012) } \\
{[37]}\end{array}$ & 116 & $x$ & $72.4 \%$ & $\begin{array}{l}\text { Nurses, } \\
\text { physicians and } \\
\text { assistants }\end{array}$ & Transversal & $\begin{array}{l}\text {-Maslach Burnout } \\
\text { Inventory (MBI) }\end{array}$ & -Mobbing Questionnaire & $\begin{array}{l}\text {-Job satisfaction questionnaire. } \\
\text {-Work commitment }\end{array}$ \\
\hline $\begin{array}{l}\text { (Roldán, 2012) } \\
\text { [38] }\end{array}$ & 315 & 43.92 & $37.4 \%$ & Physicians & Transversal & $\begin{array}{l}\text {-Maslach Burnout } \\
\text { Inventory (MBI) }\end{array}$ & $\begin{array}{l}\text {-Questionnaire on the } \\
\text { frequency of assaults } \\
\text { suffered at work. }\end{array}$ & $\begin{array}{l}\text {-The Beck Depression Inventory (BDI). } \\
\text {-The Beck Anxiety Inventory (BAI). }\end{array}$ \\
\hline $\begin{array}{l}\text { (Spence-Laschinger, } \\
\text { 2012) } \\
{[39]}\end{array}$ & 205 & $x$ & $92 \%$ & Nurses & Transversal & $\begin{array}{l}\text {-Maslach Burnout } \\
\text { Inventory (MBI) }\end{array}$ & $\begin{array}{l}\text {-Questionnaire on the } \\
\text { frequency of assaults } \\
\text { suffered at work. }\end{array}$ & -Work satisfaction (Shaver \& Lacey, 2003). \\
\hline $\begin{array}{l}\text { (Galián-Muñoz, } \\
\text { 2013) } \\
\text { [40] }\end{array}$ & 137 & 42 & $82.5 \%$ & $\begin{array}{l}\text { Nurses and } \\
\text { assistants }\end{array}$ & Transversal & $\begin{array}{l}\text {-Maslach Burnout } \\
\text { Inventory (MBI) }\end{array}$ & $\begin{array}{l}\text { Hospital Aggressive } \\
\text { Behaviour Scale-Users }\end{array}$ & -General Health Questionnaire GHQ-28 \\
\hline
\end{tabular}


Table 1. Cont.

\begin{tabular}{|c|c|c|c|c|c|c|c|c|}
\hline Autores & $n$ & Age & \% Women & Type Work & Type Study & Burnout Evaluation & $\begin{array}{l}\text { Workplace Violence } \\
\text { Evaluation }\end{array}$ & Otras Medidas \\
\hline $\begin{array}{l}\text { (Read, 2013) } \\
\quad[41]\end{array}$ & 342 & 28.1 & $91.5 \%$ & Nurses & Transversal & $\begin{array}{l}\text {-Maslach Burnout } \\
\text { Inventory (MBI) }\end{array}$ & $\begin{array}{l}\text {-Negative Acts } \\
\text { Questionnaire-- } \\
\text { Revised (NAQ-R) } \\
\text {-Workplace Incivility Scale } \\
\text { (Cortina et al. 2001). }\end{array}$ & $\begin{array}{l}\text { Conditions of Work Effectiveness Questionnaire } \\
\text { II (Laschinger et al. 2001) } \\
\text { Career Turnover Intentions (Kelloway et al., } \\
\text { 1999) } \\
\text {-Areas of Worklife Scale, Community subscale } \\
\text { (Leiter and Maslach, 2004) } \\
\text {-Areas of Worklife Scale, Values subscale (Leiter } \\
\text { and Maslach, 2004) } \\
\text {-Areas of Worklife Scale, Fairness subscale } \\
\text { (Leiter and Maslach, 2004) } \\
\text {-Psychological Capital Questionnaire (Leiter and } \\
\text { Maslach, 2004) } \\
\text {-Authentic Leadership Questionnaire (Luthans } \\
\text { et al., 2007) } \\
\text {-Pressure Management IndicatorPhysical } \\
\text { Symptoms subscaleEnergy Levels subscale } \\
\text { (Williams and Cooper, 1998) }\end{array}$ \\
\hline $\begin{array}{c}\text { (Pineau-Stamr, 2013) } \\
\text { [42] }\end{array}$ & 205 & 28.1 & $91.5 \%$ & Nurses & Longitudinal & $\begin{array}{l}\text {-Maslach Burnout } \\
\text { Inventory (MBI) }\end{array}$ & $\begin{array}{l}\text {-Negative Acts } \\
\text { Questionnaire-- } \\
\text { Revised (NAQ-R) }\end{array}$ & $\begin{array}{l}\text {-Job Turnover Intentions } \\
\text { Scale, (Kelloway, Gottlieb, y Barham's 1999). } \\
\text {-Authentic Leadership Questionnaire (ALQ) }\end{array}$ \\
\hline $\begin{array}{l}\text { (Threadgill, 2013) } \\
{[43]}\end{array}$ & 185 & $x$ & $86 \%$ & Nurses & Transversal & $\begin{array}{l}\text {-Maslach Burnout } \\
\text { Inventory (MBI) }\end{array}$ & $\begin{array}{l}\text {-Negative Acts } \\
\text { Questionnaire-Revised } \\
\text { (NAQ-R) }\end{array}$ & $\begin{array}{l}\text {-Demographic and professional background. } \\
\text {-intention to quit }\end{array}$ \\
\hline $\begin{array}{c}\text { (Hensel, 2014) } \\
\text { [44] }\end{array}$ & 671 & 38.7 & $83.0 \%$ & Assistants & Transversal & $\begin{array}{l}\text { Maslach Burnout } \\
\text { Inventory-Human } \\
\text { Services Survey } \\
\text { (MBI-HSS) }\end{array}$ & $\begin{array}{l}\text {-Staff Observation } \\
\text { Assessment Scale-revised } \\
\text { (SOAS-R). } \\
\text {-Questionnaire on the } \\
\text { frequency of assaults } \\
\text { suffered at work. }\end{array}$ & $\begin{array}{l}\text {-Emotional reactions to aggressive behaviour } \\
\text { scale. } \\
\text {-Difficult behaviour self-efficacy scale. } \\
\text {-Positive Work Motivations Scale (PWMS) and } \\
\text { General Positive Contributions Scale (GPCS). }\end{array}$ \\
\hline $\begin{array}{c}(\mathrm{Hu}, 2014) \\
{[45]}\end{array}$ & 424 & $x$ & $96 \%$ & Nurses & Transversal & $\begin{array}{l}\text {-Maslach Burnout } \\
\text { Inventory (MBI) }\end{array}$ & $\begin{array}{l}\text { Own questionnaire on } \\
\text { assaults suffered at work. }\end{array}$ & -Demographic and professional background. \\
\hline $\begin{array}{c}\text { (Trépanier, 2014) } \\
{[46]}\end{array}$ & 699 & 43.99 & $90.5 \%$ & Nurses & Longitudinal & $\begin{array}{l}\text {-Maslach Burnout } \\
\text { Inventory General } \\
\text { Survey (MBI-GS) }\end{array}$ & $\begin{array}{l}\text { Negative Acts } \\
\text { Questionnaire- } \\
\text { Revised (NAQ-R) }\end{array}$ & $\begin{array}{l}\text {-Satisfaction: Work-Related Basic Need } \\
\text { Satisfaction scale }\end{array}$ \\
\hline
\end{tabular}


Table 1. Cont

\begin{tabular}{|c|c|c|c|c|c|c|c|c|}
\hline Autores & $n$ & Age & $\%$ Women & Type Work & Type Study & Burnout Evaluation & $\begin{array}{l}\text { Workplace Violence } \\
\text { Evaluation }\end{array}$ & Otras Medidas \\
\hline $\begin{array}{l}\text { (Waschgler, 2014) } \\
{[19]}\end{array}$ & 694 & 42 & $83.4 \%$ & Nurses & Transversal & $\begin{array}{l}\text {-Maslach Burnout } \\
\text { Inventory (MBI) }\end{array}$ & Overall Job Satisfaction & -General Health Questionnaire GHQ-28. \\
\hline $\begin{array}{l}\text { (Alameddine, 2015) } \\
\text { [48] }\end{array}$ & 915 & $x$ & $79.4 \%$ & Nurses & Transversal & $\begin{array}{l}\text {-Maslach Burnout } \\
\text { Inventory (MBI) }\end{array}$ & $\begin{array}{l}\text {-Exposure and consequences } \\
\text { of violence: verbal abuse and } \\
\text { physical violence in the last } \\
12 \text { months }\end{array}$ & $\begin{array}{l}\text {-Demographic and professional background. } \\
\text {-Intention to quit }\end{array}$ \\
\hline $\begin{array}{l}\text { (Dal-Pai, 2015) } \\
\text { [49] }\end{array}$ & 269 & 48.5 & $73 \%$ & $\begin{array}{l}\text { Nurses and } \\
\text { Physicians }\end{array}$ & Transversal & $\begin{array}{l}\text {-Maslach Burnout } \\
\text { Inventory (MBI) }\end{array}$ & $\begin{array}{l}\text {-Survey Questionnaire } \\
\text { Workplace Violence in the } \\
\text { Health Sector }\end{array}$ & -Self-Report Questionnaire (SRQ-20) \\
\hline $\begin{array}{c}\text { (Menon, 2015) } \\
{[50]}\end{array}$ & 99 & 40 & $50 \%$ & $\begin{array}{l}\text { Nurses and } \\
\text { Physicians }\end{array}$ & Transversal & $\begin{array}{l}\text {-Maslach Burnout } \\
\text { Inventory (MBI) }\end{array}$ & $\begin{array}{l}\text {-Descriptive questionnaire on } \\
\text { working conditions and } \\
\text { violence suffered. }\end{array}$ & Work stress evaluation questionnaire. \\
\hline $\begin{array}{c}\text { (Pintado-Cucarella, } \\
\text { 2015) } \\
{[51]}\end{array}$ & 29 & 39.24 & $65.5 \%$ & Physicians & Transversal & $\begin{array}{l}\text { Maslach Burnout } \\
\text { Inventory-Human } \\
\text { Services Survey } \\
\text { (MBI-HSS) }\end{array}$ & $\begin{array}{l}\text { Own questionnaire on } \\
\text { assaults suffered at work }\end{array}$ & Jefferson Medical Empathy Scale \\
\hline $\begin{array}{c}\text { (Viotti, 2015) } \\
{[52]}\end{array}$ & 630 & 37.97 & $82.2 \%$ & $\begin{array}{l}\text { Nurses and } \\
\text { Assistants }\end{array}$ & Transversal & $\begin{array}{l}\text {-Maslach Burnout } \\
\text { Inventory (MBI) }\end{array}$ & $\begin{array}{l}\text {-Customer-Related Social } \\
\text { stressors (CSS) inventory }\end{array}$ & -Labor resources. \\
\hline $\begin{array}{c}\text { (Deniz, 2016) } \\
{[53]}\end{array}$ & 120 & 29.47 & $46.7 \%$ & $\begin{array}{l}\text { Nurses and } \\
\text { Physicians }\end{array}$ & Transversal & $\begin{array}{l}\text {-Maslach Burnout } \\
\text { Inventory (MBI) }\end{array}$ & $\begin{array}{l}\text { Own questionnaire on } \\
\text { assaults suffered at work. }\end{array}$ & Demographic and professional background. \\
\hline $\begin{array}{l}\text { (Galián-Muñoz, } \\
\text { 2016) } \\
\text { [54] }\end{array}$ & 1489 & 42.09 & $82.7 \%$ & $\begin{array}{l}\text { Nurses, } \\
\text { physicians and } \\
\text { assistants }\end{array}$ & Transversal & $\begin{array}{l}\text {-Maslach Burnout } \\
\text { Inventory (MBI) }\end{array}$ & $\begin{array}{l}\text { The Hospital Aggressive } \\
\text { Behaviour Scale-Users } \\
\text { (HABS-U). }\end{array}$ & -Job satisfaction. \\
\hline $\begin{array}{c}\text { (Llor-Esteban, 2016) } \\
{[55]}\end{array}$ & 518 & 41.3 & $86.3 \%$ & Nurses & Transversal & $\begin{array}{l}\text {-Maslach Burnout } \\
\text { Inventory-General } \\
\text { Survey (MBI-GS). }\end{array}$ & $\begin{array}{l}\text { Own questionnaire on } \\
\text { assaults suffered at work }\end{array}$ & -Overall Job Satisfaction Scale (OJS). \\
\hline $\begin{array}{c}\text { (Anwar, 2017) } \\
{[56]}\end{array}$ & 286 & 28.9 & $x$ & Nurses & Transversal & $\begin{array}{l}\text {-Maslach Burnout } \\
\text { Inventory (MBI) }\end{array}$ & $\begin{array}{l}\text { Own questionnaire on } \\
\text { assaults suffered at work. }\end{array}$ & Demographic and professional background. \\
\hline
\end{tabular}


Table 1. Cont.

\begin{tabular}{|c|c|c|c|c|c|c|c|c|}
\hline Autores & $n$ & Age & \% Women & Type Work & Type Study & Burnout Evaluation & $\begin{array}{c}\text { Workplace Violence } \\
\text { Evaluation }\end{array}$ & Otras Medidas \\
\hline $\begin{array}{c}\text { (Bawakid, 2017) } \\
\text { [57] }\end{array}$ & 246 & 35 & $57.3 \%$ & Physicians & Transversal & $\begin{array}{l}\text {-Maslach Burnout } \\
\text { Inventory (MBI) }\end{array}$ & $\begin{array}{l}\text { Own questionnaire on } \\
\text { assaults suffered at work. }\end{array}$ & Demographic and professional background. \\
\hline $\begin{array}{c}\text { (Hamdan, 2017) } \\
\text { [59] }\end{array}$ & 444 & 30 & $76.8 \%$ & $\begin{array}{l}\text { Nurses and } \\
\text { Physicians }\end{array}$ & Transversal & $\begin{array}{l}\text {-Maslach Burnout } \\
\text { Inventory (MBI) }\end{array}$ & $\begin{array}{l}\text { Own questionnaire on } \\
\text { assaults suffered at work. }\end{array}$ & Demographic and professional background. \\
\hline $\begin{array}{c}\text { (Rafeea, 2017) } \\
{[61]}\end{array}$ & 100 & 35 & $66 \%$ & $\begin{array}{c}\text { Nurses, } \\
\text { Physicians and } \\
\text { Assistants }\end{array}$ & Transversal & $\begin{array}{l}\text {-Maslach Burnout } \\
\text { Inventory (MBI) }\end{array}$ & $\begin{array}{l}\text { Own questionnaire on } \\
\text { assaults suffered at work }\end{array}$ & -Demographic and professional background. \\
\hline $\begin{array}{l}\text { (Wongtongkam, } \\
\text { 2017) } \\
{[62]}\end{array}$ & 48 & 39.19 & $64.8 \%$ & Nurses & Transversal & $\begin{array}{l}\text {-Abbreviated Maslach } \\
\text { Burnout Inventory. }\end{array}$ & $\begin{array}{l}\text { Workplace violence: personal } \\
\text { experience of the last } 30 \text { days, } \\
\text { causes and contributing } \\
\text { factors. } \\
\text { Authors' characteristics }\end{array}$ & $\begin{array}{l}\text { Available resources. } \\
\text { Impact of event scale-revised (IES-R). Evaluate } \\
\text { Post-Traumatic Stress. }\end{array}$ \\
\hline $\begin{array}{c}\text { (Copeland, 2018) } \\
\text { [63] }\end{array}$ & 147 & 35 & $63 \%$ & $\begin{array}{l}\text { Nurses and } \\
\text { physicians }\end{array}$ & Transversal & $\begin{array}{l}\text {-Maslach Burnout } \\
\text { Inventory (MBI) }\end{array}$ & $\begin{array}{l}\text { Own questionnaire on } \\
\text { assaults suffered at work. }\end{array}$ & -Job Satisfaction \\
\hline $\begin{array}{c}\text { (Coskun-Cenk, 2018) } \\
\text { [64] }\end{array}$ & 143 & $\mathrm{x}$ & $58.7 \%$ & $\begin{array}{l}\text { Nurses and } \\
\text { physicians }\end{array}$ & $\begin{array}{l}\text { Transversal- } \\
\text { descriptive }\end{array}$ & $\begin{array}{l}\text {-Maslach Burnout } \\
\text { Inventory (MBI) }\end{array}$ & $\begin{array}{l}\text { Own questionnaire on } \\
\text { assaults suffered at work. }\end{array}$ & Demographic and professional background. \\
\hline $\begin{array}{c}(\mathrm{Kim}, 2018) \\
{[65]}\end{array}$ & 170 & $\mathrm{x}$ & $75 \%$ & Nurses & Transversal & $\begin{array}{l}\text {-Maslach Burnout } \\
\text { Inventory (MBI) }\end{array}$ & $\begin{array}{l}\text { Own questionnaire on } \\
\text { assaults suffered at work. }\end{array}$ & $\begin{array}{l}\text { AACN's HWE Questionnaire: Evaluates } \\
\text { collaboration between colleagues, } \\
\text { communication skills, effectiveness of } \\
\text { decision-making, leadership, recognition }\end{array}$ \\
\hline $\begin{array}{c}\text { (Looff, 2018) } \\
{[66]}\end{array}$ & 114 & 35.2 & $59 \%$ & Nurses & Transversal & $\begin{array}{l}\text {-Maslach Burnout } \\
\text { Inventory (MBI) }\end{array}$ & $\begin{array}{l}\text {-Modified Overt Aggression } \\
\text { Scale (Oliver, Crawford, Rao, } \\
\text { Reece, \& Tyrer, 2007). }\end{array}$ & $\begin{array}{l}\text {-Dutch Bar-On Emotional Quotient Inventory. } \\
\text { Bar-On 2006): Evaluate Emotional Intelligence. } \\
\text {-NEO Five-Factor Inventory (NEO-FFI): } \\
\text { Evaluate personality factors. } \\
\text {-Demands and Support questionnaire: Assess } \\
\text { emotional stress. }\end{array}$ \\
\hline
\end{tabular}


Table 1. Cont

\begin{tabular}{|c|c|c|c|c|c|c|c|c|}
\hline Autores & $n$ & Age & $\%$ Women & Type Work & Type Study & Burnout Evaluation & $\begin{array}{l}\text { Workplace Violence } \\
\text { Evaluation }\end{array}$ & Otras Medidas \\
\hline $\begin{array}{l}\text { (Shier, 2018) } \\
\quad[67]\end{array}$ & 674 & 42.91 & $86.6 \%$ & $\begin{array}{c}\text { Nurses, } \\
\text { Physicians and } \\
\text { Assistants }\end{array}$ & Transversal & $\begin{array}{l}\text {-Maslach Burnout } \\
\text { Inventory (MBI) }\end{array}$ & $\begin{array}{l}\text { Own questionnaire on } \\
\text { assaults suffered at work. }\end{array}$ & $\begin{array}{l}\text {-Relationships } \\
\text {-Intention to Leave-A further adaptation to } \\
\text { Kline and Graham (2009): intention to quit work. } \\
\text {-Life Scale (SWLS): Satisfaction with life. }\end{array}$ \\
\hline $\begin{array}{l}\text { (Zaczyk, 2018) } \\
{[68]}\end{array}$ & 74 & 40 & $90.5 \%$ & Nurses & Transversal & $\begin{array}{l}\text {-Maslach Burnout } \\
\text { Inventory (MBI) }\end{array}$ & $\begin{array}{l}\text { Own questionnaire on } \\
\text { assaults suffered at work }\end{array}$ & Demographic and professional background. \\
\hline $\begin{array}{c}\text { (Aguilar-Nájera, } \\
\text { 2019) } \\
\text { [69] }\end{array}$ & 411 & $x$ & $x$ & Physicians & Transversal & $\begin{array}{l}\text {-Maslach Burnout } \\
\text { Inventory (MBI) }\end{array}$ & $\begin{array}{l}\text { Own questionnaire on } \\
\text { assaults suffered at work }\end{array}$ & Demographic and professional background. \\
\hline $\begin{array}{l}\text { (Ajoudani, 2019) } \\
{[70]}\end{array}$ & 278 & 33.76 & $85.8 \%$ & Nurses & Transversal & $\begin{array}{l}\text {-Maslach Burnout } \\
\text { Inventory (MBI) }\end{array}$ & $\begin{array}{l}\text {-Negative Acts Questionnaire } \\
\text { (NAQ) }\end{array}$ & -Moral Distress Scale-Revised \\
\hline $\begin{array}{c}(\text { Akram, 2019) } \\
{[71]}\end{array}$ & 350 & 42.6 & $40 \%$ & Physicians & Transversal & $\begin{array}{l}\text { Maslach Burnout } \\
\text { Inventory-General } \\
\text { Survey (MBI-GS). }\end{array}$ & $\begin{array}{l}\text {-Counterproductive Work } \\
\text { Behavior } \\
\text {-Abusive supervisor. }\end{array}$ & -Labor resources. \\
\hline $\begin{array}{l}\text { (Andela,2018) } \\
{[72]}\end{array}$ & 481 & 39 & $96 \%$ & Nurses & Transversal & $\begin{array}{l}\text {-Maslach Burnout } \\
\text { Inventory (MBI) }\end{array}$ & $\begin{array}{l}\text { Elderly abuse scale } \\
\text { developed by Huguenotte } \\
\text { (2012). }\end{array}$ & $\begin{array}{l}\text {-Job stressors and job resources were measured } \\
\text { scale. }\end{array}$ \\
\hline $\begin{array}{c}\text { (Castro Negreiros, } \\
\text { 2019) } \\
{[74]}\end{array}$ & 96 & $x$ & $x$ & Assitants & Transversal & $\begin{array}{l}\text {-Maslach Burnout } \\
\text { Inventory (MBI) }\end{array}$ & -Test Escala Cisneros. & Demographic and professional background. \\
\hline $\begin{array}{c}\text { (Goussinsky, 2019) } \\
\text { [75] }\end{array}$ & 105 & 37.7 & $84 \%$ & Nurses & Transversal & $\begin{array}{l}\text {-Maslach Burnout } \\
\text { Inventory (MBI) }\end{array}$ & $\begin{array}{l}\text {-Own questionnaire on } \\
\text { assaults suffered at work. }\end{array}$ & $\begin{array}{l}\text {-Coworker support Sub-scale: Peer support. } \\
\text {-Occupational Coping } \\
\text { Self-EfficacyQuestionnaire for Nurses } \\
\text { (OCSE-N): Self-efficacy strategies. }\end{array}$ \\
\hline $\begin{array}{l}\text { (Jiménez, 2019) } \\
{[76]}\end{array}$ & 565 & 36 & 37.4 & $\begin{array}{l}\text { Nurses and } \\
\text { Physicians }\end{array}$ & Transversal & $\begin{array}{l}\text {-Maslach Burnout } \\
\text { Inventory (MBI) }\end{array}$ & $\begin{array}{l}\text {-Own questionnaire on } \\
\text { assaults suffered at work. }\end{array}$ & Demographic and professional background. \\
\hline $\begin{array}{l}\text { (Looff, 2019) } \\
\quad[77]\end{array}$ & 110 & 35.5 & $59 \%$ & Nurses & Longitudinal & $\begin{array}{l}\text {-Maslach Burnout } \\
\text { Inventory (MBI) }\end{array}$ & $\begin{array}{l}\text { Questionnaire on the } \\
\text { frequency of violence } \\
\text { suffered at work. }\end{array}$ & $\begin{array}{l}\text { Dutch Bar-On Emotional Quotient Inventory. } \\
\text { Bar-On 2006): Evaluate Emotional Intelligence. } \\
\text {-NEO Five-Factor Inventory (NEO-FFI): } \\
\text { Evaluate personality factors. } \\
\text {-Demands and Support questionnaire: Assess } \\
\text { emotional stress. }\end{array}$ \\
\hline
\end{tabular}


Table 1. Cont

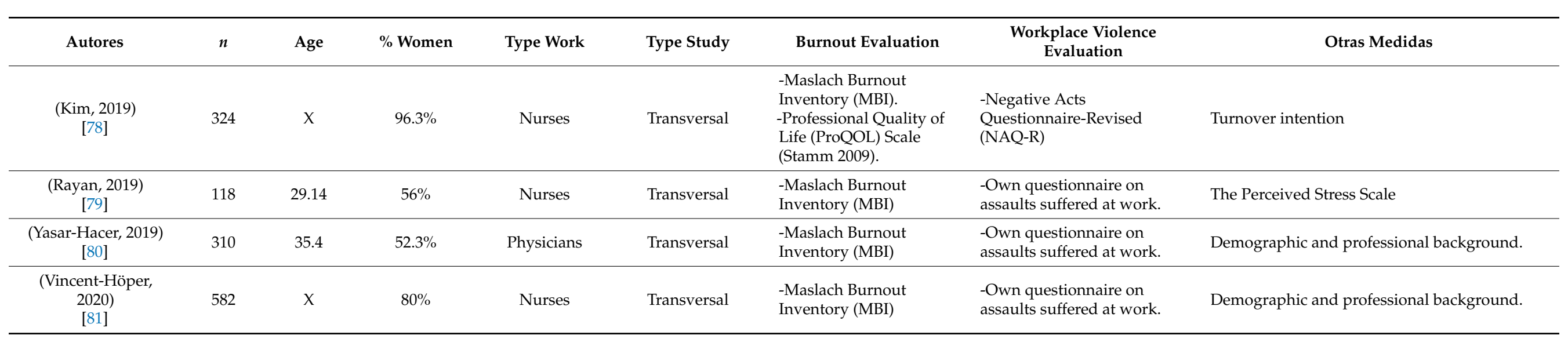


Table 2. Descriptive results.

\begin{tabular}{cccc}
\hline Variable & Categories & N (=22,993) & \% \\
\hline Gender & Women & 14,126 & 61.43 \\
& Men & 8867 & 38.56 \\
Profession & Nurses & 16,473 & 71.64 \\
& Physicians & 6551 & 28.49 \\
& Assistants & 269 & 1.16 \\
Specialty & ICU & 9344 & 40.63 \\
& Emergency & 6719 & 29.22 \\
& Mental Health & 4236 & 18.54 \\
& Surgery & 4187 & 18.45 \\
& Paediatrics & 2080 & 9.04 \\
& Internal Medicine & 1552 & 6.74 \\
& Admissions & 915 & 3.97 \\
& & & \\
& Full-Time & 13258 & 57.66 \\
Academic level & Part-Time & 6456 & 28.07 \\
& Temporary & 3279 & 14.2 \\
& University degree & 8793 & 38.02 \\
& Vocational training/technicians & 7512 & 32.67 \\
& Secondary school certificate & 6642 & 28.88 \\
& PhD & 46 & 0.20
\end{tabular}

Table 3 (See Table 3) shows the results of the mean of the different levels that make up burnout.

Table 3. Results of the mean of the different levels that make up burnout.

\begin{tabular}{|c|c|c|c|c|}
\hline \multirow[t]{2}{*}{ Variable } & \multirow[t]{2}{*}{ Category } & \multicolumn{3}{|c|}{ Mean (SD) of the Subscales Created by the MBI } \\
\hline & & $\mathrm{EE}$ & DP & PA \\
\hline \multicolumn{5}{|l|}{ Gender } \\
\hline & Men & $28.2(6.0)$ & $12.2(4.2)$ & $24.3(7.2)$ \\
\hline & Women & $24.3(6.5)$ & $11.7(4.2)$ & $25.4(7.2)$ \\
\hline \multicolumn{5}{|l|}{ Profession } \\
\hline & Nurses & $28.1(6.1)$ & $11.9(4.0)$ & $25.1(6.7)$ \\
\hline & Physicians & $26.3(5.4)$ & $12.0(4.3)$ & $24.7(6.7)$ \\
\hline \multicolumn{5}{|l|}{ Specialty } \\
\hline & ICU & $29.1(6.4)$ & $12.8(3.9)$ & $27.7(6.3)$ \\
\hline & Emergency & $29.6(6.1)$ & $10.8(4.1)$ & $25.4(7.3)$ \\
\hline & Mental Health & $28.0(6.5)$ & $12.1(3.9)$ & $28.6(5.9)$ \\
\hline & Surgery & $27.7(6.7)$ & $12.6(4.1)$ & $27.4(5.8)$ \\
\hline & Paediatrics & $24.1(6.4)$ & $11.1(3.8)$ & $24.6(5.2)$ \\
\hline & Internal Medicine & $26.3(6.6)$ & $10.2(3.3)$ & $26.8(6.7)$ \\
\hline & Admissions & $24.4(6.8)$ & $9.8(4.1)$ & $1.9(3.9)$ \\
\hline \multicolumn{5}{|c|}{ Type of employment } \\
\hline & Full-time & $28.3(7.4)$ & $12.8(4.1)$ & $28.4(6.3)$ \\
\hline & Part-time & $24.1(6.8)$ & $10.2(4.4)$ & $23.2(4.6)$ \\
\hline & Temporary & $27.6(7.1)$ & $11.9(3.9)$ & $25.6(6.9)$ \\
\hline \multicolumn{5}{|l|}{ Experience } \\
\hline & Less than 5 years & $28.8(6.3)$ & $12.2(4.0)$ & $23.5(6.3)$ \\
\hline & 5-15 years & $28.2(6.3)$ & $11.3(3.3)$ & $25.4(7.7)$ \\
\hline & $15-30$ years & $25.9(6.5)$ & $12.7(4.3)$ & $25.2(6.4)$ \\
\hline & More than 30 years & $26.4(6.7)$ & $11.7(4.2$ & $23.5(6.4)$ \\
\hline
\end{tabular}


Regarding gender, we can observe that women in the health sector appear to suffer from higher burnout levels than men. For example, women $(\bar{x}=28.2 ; S D=6.0)$ seem to have higher levels of EE compared to men $(\bar{x}=24.3 ; S D=6.5)$ and the same hold with DP, in which women get a score of $(\bar{x}=12.2 ; S D=4.2)$, while that for men is $(\bar{x}=11.7 ; S D=4.2)$. The PA subcategory is the only variable in which men $(\bar{x}=24.3 ; S D=7.2)$ obtain a higher score than women $(\bar{x}=25.4 ; S D=7.2)$. Regarding the professional category, it is nurses who obtain the worst results in terms of $\operatorname{EE}(\bar{x}=28.1 ; S D=6.1)$ to DP $(\bar{x}=11.9 ; S D=4.0)$ and PA $(\bar{x}=25.1 ; S D=6.7)$. In the specialty area, there are three categories with very similar values, including the Intensive Care Unit (ICU) with an EE of $(\bar{x}=29.1 ; S D=6.4)$, a DP of $(\bar{x}=12.8 ; S D=3.9)$ and a PA of $(\bar{x}=27.7 ; S D=6.3)$. The Emergency specialty has an EE of $(\bar{x}=29.6 ; S D=6.1)$, a DP of $(\bar{x}=10.8 ; S D=4.1)$ and a PA of $(\bar{x}=25.4 ; S D=7.3)$. Similar values were found in the Mental Health specialty, with an EE of $(\bar{x}=28.0 ; S D=6.5)$, a DP of $(\bar{x}=12.1 ; S D=3.9)$ and a PA of $(\bar{x}=28.6 ; S D=5.9)$.

Table 4 (See Table 4 ) shows the main characteristics of violence and the protective and risk factors that promote it.

Table 4. Main characteristics of the violence suffered at workplace and the protective and risk factors.

\begin{tabular}{|c|c|c|c|c|}
\hline Variable & Categories & Subcategories & $N=72$ & $\%$ \\
\hline \multicolumn{5}{|c|}{ Causal Agent } \\
\hline & Patient & & 65 & $90.2 \%$ \\
\hline & $\begin{array}{c}\text { Partner/Family } \\
\text { Members }\end{array}$ & & 26 & $36.1 \%$ \\
\hline & Coworkers & & 11 & $15.2 \%$ \\
\hline \multicolumn{5}{|c|}{ Type of violence } \\
\hline & Verbal & & 72 & $100 \%$ \\
\hline & Physical & & 57 & $79.1 \%$ \\
\hline & Sexual & & 23 & $31.9 \%$ \\
\hline \multirow{2}{*}{\multicolumn{5}{|c|}{ Risk and protective factors }} \\
\hline & & & & \\
\hline & & Full-Time & 48 & $66.6 \%$ \\
\hline & & Part-Time & 36 & $52 \%$ \\
\hline & & Social Support & 70 & $97.2 \%$ \\
\hline & & Family Support & 35 & $48.6 \%$ \\
\hline & & Quality of the work environment & 17 & $23.6 \%$ \\
\hline & & Leadership & 22 & $30.5 \%$ \\
\hline & & Level of Autonomy & 9 & $12.5 \%$ \\
\hline & & Resource Access & 25 & $34.7 \%$ \\
\hline \multicolumn{5}{|c|}{ Personal } \\
\hline & & Job satisfaction & 70 & $97.2 \%$ \\
\hline & & Empathy & 11 & $15.2 \%$ \\
\hline & & Adaptation & 7 & $9.7 \%$ \\
\hline & & Coping strategies & 28 & $38.8 \%$ \\
\hline & & Self-efficacy & 12 & $16.6 \%$ \\
\hline
\end{tabular}

The vast majority of the studies analysed $(N=65 ; \%=90.2 \%)$ show that the most frequent causal agent endured by healthcare professionals is the patient; to a lesser extent, family members or partners $(N=26 ; \%=36.1 \%)$; and finally, coworkers $(N=11 ; \%=15.2 \%)$. The most common type of violence, as collected by all the investigations, are verbal ( $N=72$; $\%=100 \%$ ), including shouting, insults or threats. Workers claim that at some time in their working life they have suffered some type of physical violence $(N=57 ; \%=79.1 \%)$ and, to a lesser extent, a sexual assault $(N=23 ; \%=31.9 \%)$. Regarding risk and protective factors, it should be clarified that any action contrary to a risk factor, or that goes in the opposite direction, is understood as a protective factor. Thus, the factors were classified into two series of categories, the structural/organizational type, and the personal type. For example, within the former group, $97 \%(N=70)$ of the studies analysed found that social support from coworkers is one of the risk and protective factors that most affect workplace violence 
in the healthcare sector; this would also be highly influenced by family support $(N=35$; $\%=48.6 \%)$. A determining factor seems to be the professionals' type of contract, full-time $(N=48 ; \%=66.6 \%)$ or part-time $(N=36 ; \%=52 \%)$. The quality of the work environment $(N=17 ; \%=23.6 \%)$, the type of leadership of the plant manager $(N=22 ; \%=30.5 \%)$ and the level of autonomy $(N=9 ; \%=12.5)$ are among the other risk and protective factors found. On the other hand, personal factors are those related, for example, to workers' job satisfaction $(N=70 ; \%=97.2 \%)$, coping strategies $(N=28 ; \%=38.8 \%)$, self-efficacy $(N=12$; $\%=16.6 \%)$ or empathy $(N=11 ; \%=15.2 \%)$.

\section{Discussion}

The objective of this systematic review is to observe the levels of WV and burnout in the healthcare sector and to verify the most influential risk and protective factors.

The analysis of the results of the different studies finally selected shows that there is a high percentage of professionals who at some time in their working lives have been exposed to some type of $\mathrm{WV}$, whether verbal, physical, or sexual. This, in turn, appears to affect the high levels of observed burnout, which are higher among women than among men, although this may also be due to the large female representation in the total sample. Following in this same line, according to the Labour Force Survey (LFS) developed by the National Institute of Statistics (INE, from its Spanish initials) in Spain, with data from the last quarter of 2018 , women appear to hold $71.7 \%$ of jobs in the healthcare sector [82]. Among many other variables, the burnout of professionals is mainly influenced by their specialization, type of work employment and their experience within the field. When it comes to the type of contract, full-time employment involves more effort on the part of the worker and requires greater attitudinal resources to carry out the work required, these cause greater emotional exhaustion (EE), lower feelings of personal accomplishment (PA) and therefore more negative thoughts about their competency (DP) [28].

In relation to the profession among nurses and doctors that suffers the greatest $\mathrm{WV}$, the nursing profession faces the most exposure. This is due, in large part, to the fact that they are directly in contact with patients. They are, therefore, the first exposed to complicated situations that require a lot of control and that generate great emotional wear. Various studies have shown that it would be the nurses who, due to the situations of experienced violence, would obtain the worst levels of burnout and emotional intelligence. This would translate into high levels of anxiety, lack of self-esteem, insomnia problems, depression or even physical effects such as a higher percentage of those suffering from cancer. [83]

The specialties in which higher levels of burnout are contemplated are actually those in which patients are in the most serious conditions and which require more need to manage unpredictability in the workplace, as can happen in the Intensive Care Unit (ICU), in the emergency room, or in the mental health area, which involves unpredictable patients with low levels of self-control [84]. These studies corroborate that high levels of burnout in specializations where the patient's life may be in danger correlate with a high percentage of WV [85], mainly from the patients, family members or partners. It can be seen that despite the pressure affecting nurses, doctors and nursing assistants, among others, there is not a high percentage of attacks on the part of coworkers.

There are many risk factors that make WV more likely. If one examines them indepth, what is striking is that those with the most impact are related to high levels of effort (structural and/or organizational factors) and workers' lack of self-control and selfregulation skills (personal factors). Two types of factors were categorized for a better understanding, but neither should be understood separately, but rather as a set that affects and influences everyone equally. For example, a healthcare professional who has a fulltime work contract is more likely to be exposed to WV, since this entails greater emotional exhaustion, and more exposure to extreme situations and to all types of people with multiple reactions. We understand, in accordance with the results, that this probability of experiencing WV will increase if he or she works within a specialty in which moments of great tension and stress are experienced. However, in relation to this equation, various 
types of factors, both organizational and personal, can considerably reduce the WV suffered, such as a healthy work environment, high levels of social support from colleagues, or even possessing intrinsic coping strategy, self-efficacy and adaptation resources. These, in turn, would increase job satisfaction and therefore reduce the frequency of WV [86], causing burnout levels to decrease drastically, with the health benefits this environment entails [87].

It is here, therefore, that the role of institutions becomes important. Creating intervention programmes that aim to alleviate levels of WV should be a priority. For example, the Zero Tolerance Policy is a political campaign, spread all over the world, a benchmark in the United Kingdom that includes several organizations such as the British Columbia Occupational Health and the Safety Agency for Healthcare and the National Health Service (NHS) [30], and addresses the issue of violence against healthcare workers on the part of co-workers and beneficiaries. This campaign provides healthcare personnel with the necessary resources, such as courses or workshops and the creation of support networks within the community, and promotes awareness in relation to patients to show the reality of this matter. For this, a positive work culture must be created where all those involved (workers and patients) treat each other with respect, with a focus on positive work recognition and conflict resolution [88]. The application of these types of policies can have a great benefit for workers, such as a more positive responses to stressful situations [61].

Another very similar program was the Assaulted Staff Action Program (ASAP), which aimed to identify "high-risk" patients (with a criminal record or previous reports of high levels of aggression) and to give workers suitable warning prior to the visit. According to some studies, it was shown that WV decreased significantly when using this method [88]. In that sense, it is important to note that workplace violence is one of the possible causes of burnout in the health sector [81].

Therefore, this is not merely an internal struggle on the part of workers when facing patients. Government agencies should also be the main stakeholders and work alongside other parties to reduce such incidents, as the quality of the healthcare sector depends on the satisfaction of nurses, doctors and other specialized employees.

\section{Limitations and Future Research}

The present systematic review has some limitations. The inclusion and exclusion criteria were clearly defined, but more research into a more specific database such as CINAHL (Cumulative Index to Nursing \& Allied Health Literature) would be advised in the case whereby an article only appears in it and not in WOS. There is also the risk of an article included in the so-called gray literature (doctoral theses, etc.) that was not located.

It would be interesting in future studies to focus on examining other variables considered relevant in the selected studies on psychoeducational intervention programs in which this instrument is applied. In this sense, this study can provide useful information to researchers and professionals for decision-making in this thematic line. More longitudinal research is required focused on evaluating the effectiveness of programs for the prevention of burnout and that not only focus on studying the effects of burnout on healthcare workers but also, for example, in the university environment. In addition, despite the importance of improving well-being and quality of life, there are several policies that could be sought to reduce the WV they experience throughout their professional lives.

\section{Conclusions}

It can be concluded that workplace violence is one of the possible causes of burnout in the health sector.

The risk and protective factors were classified into two series of categories, the structural/organizational type (for example, social support from coworkers, family support, professionals' type of contract, the quality of the work environment) and the personal type (for example, workers' job satisfaction, coping strategies, self-efficacy, empathy, etc.). Regarding risk and protective factors, it should be clarified that any action contrary to a risk factor, or that goes in the opposite direction, is understood as a protective factor. 
As we can see, there are many studies that have studied the effects of burnout on workers in the health sector, but there are few action policies that aim to reduce the WV they experience throughout their professional lives. We must emphasize that this would imply not only action from within the healthcare sector but more re-training and building awareness in society about the importance of such work, and to provide enough information to control all kinds of uncontrolled impulse in emergency situations.

We can point out the importance of institutions promoting recommendations and policies that can develop empathy and a positive work culture where those involved treat each other with respect, recognition, and work to prevent workplace violence, work stress and improve the quality of life and well-being of its members.

Author Contributions: J.M.G.L. and F.M.M.R. conceived and designed the study and contributed to the manuscript writing and data analysis. F.M.M.R., J.P.M.R. and J.M.G.L. contributed to the bibliographic review, and manuscript writing. All authors have read and agreed to the published version of the manuscript.

Funding: This research received no external funding.

Institutional Review Board Statement: Not applicable.

Informed Consent Statement: Not applicable.

Data Availability Statement: The data could be requested by the scientific community in the ethical terms to be determined.

Conflicts of Interest: The authors declare no conflict of interest.

\section{References}

1. Kobayashi, Y.; Oe, M.; Ishida, T.; Matsuoka, M.; Chiba, H.; Uchimura, N. Workplace Violence and Its Effects on Burnout and Secondary Traumatic Stress among Mental Healthcare Nurses in Japan. Int. J. Environ. Res. Public Health 2020, 17, 2747. [CrossRef]

2. Ansoleaga, E.; Gómez-Rubio, C.; Mauro, A. Violencia laboral en América Latina: Una revisión de la evidencia científica. Rev. Argent. Psiquiat. 2015, 26, 444-452.

3. Galián-Muñoz, I.; Llor-Zaragoza, P.; Ruiz-Hernández, J.A.; Jiménez-Barbero, J.A. Exposición a violencia de los usuarios y satisfacción laboral en el personal de enfermería de los hospitales públicos de la Región de Murcia. In Anales del Sistema Sanitario de Navarra; Gobierno de Navarra, Departamento de Salud: San Juan, Puerto Rico, 2018; Volume 41, pp. 181-189.

4. Neffa, J.C. Los Riesgos Psicosociales en el Trabajo; CONICET: Buenos Aires, Argentina, 2015.

5. Mucci, N.; Giorgi, G.; Cupelli, V.; Gioffre, P.A.; Rosati, M.V.; Tomei, F.; Breso-Esteve, E.; Arcangeli, G. Work-related stress assessment in a population of Italian workers. The Stress Questionnaire. Sci. Total Environ. 2015, 502, 673-679. [CrossRef]

6. Robelski, S.; Mette, J.; Wirth, T.; Kiepe, N.; Nienhaus, A.; Harth, V.; Mache, S. (Un) bounded Social Work?-Analysis of Working Conditions in Refugee and Homeless Aid in Relation to Perceived Job Stress and Job Satisfaction. Int. J. Environ. Res. Public Health 2020, 17, 601. [CrossRef]

7. Chen, S.; Lin, S.; Ruan, Q.; Li, H.; Wu, S. Workplace violence and its effect onburnout and turnover attempt among Chinese medical staff. Arch. Environ. Occup. Health 2016, 71, 330-337. [CrossRef]

8. Schaufeli, W.B.; Leiter, M.; Maslach, C. Burnout: 35 years of research and practice. Career Dev. Int. 2019, 14, 204-220. [CrossRef]

9. Maslach, C.; Jackson, S.E. The measurement of experienced burnout. J. Occup. Behav. 1981, 2, 99-113. [CrossRef]

10. Queiros, C.; Carlotto, M.S.; Kaiseler, M.; Dias, S.; Pereira, A.M. Predictors of burnout among nurses: An interactionist approach. Psicohema 2013, 25, 330-335.

11. World Health Organization. International Statistical Classification of Diseases and Related Health Problems; 10th Revision (ICD-10); World Health Organization: Geneva, Switzerland, 2012.

12. Shabsigh, R.; Rowland, D. American Psychiatric Association. Diagnostic and Statistical Manual, 4th ed.; Text Revision (DSM-IV-TR); American Psychiatric Publishing, Inc.: Washington, DC, USA, 2000.

13. Pradas-Hernández, L.; Ariza, T.; Gómez-Urquiza, J.L.; Albendín-García, L.; Emilia, I.D.1.F.; Cañadas-De la Fuente, G.A. Prevalence of burnout in paediatric nurses: A systematic review and meta-analysis. PLoS ONE 2018, 13, e0195039. [CrossRef] [PubMed]

14. Leiter, M.P.; Maslach, C. Nurse turnover: The mediating role of burnout. J. Nurs. Manag. 2009, 17, 331-339. [CrossRef]

15. Socaciu, A.I.; Ionut, R.; Barsan, M.; Ungur, A.P.; Rajnoveanu, A.M. Burnout in Gastroenterology Unit Nurses. Int. J. Environ. Res. Public Health 2020, 17, 3115. [CrossRef]

16. Ramirez-Baena, L.; Ortega-Campos, E.M.; Gomez-Urquiza, J.; Cañadas-De la Fuente, G.R.; De la Fuente-Solana, E.I.; Cañadas-De la Fuente, G.A. A multicentre study of burnout prevalence and related psychological variables in medical area hospital nurses. J. Clin. Med. 2019, 8, 92. [CrossRef]

17. O'Mahony, N. Nurse burnout and the working environment. Emerg. Nurs. 2011, 19, 30-37. [CrossRef] 
18. Gómez-Urquiza, J.L.; De la Fuente-Solana, E.I.; Albendín-García, L.; Vargas-Pecino, C.; Ortega-Campos, E.M.; Cañadas-De la Fuente, G.A. Prevalence of burnout syndrome in emergency nurses: A meta-analysis. Crit. Care Nurs. 2017, 37, e1-e9. [CrossRef]

19. Waschgler, K.; Waschgler, K.; Ruiz-Hernández, J.A.; Llor-Esteban, B.; Jiménez-Barbero, J.A. Vertical and lateral workplace bullying in nursing: Development of the hospital aggressive behaviour scale. J. Interpers. Violence 2013, 28, 2389-2412. [CrossRef]

20. Smith, D.; Cunningham, N.; Willoughby, M.; Young, C.; Odell, M.; Ibrahim, J.; Bugeja, L. The epidemiology of sexual assault of older female nursing home residents, in Victoria Australia, between 2000 and 2015. Leg. Med. 2018, 36, 89-95. [CrossRef]

21. Munn, Z.; Moola, S.; Lisy, K.; Riitano, D.; Tufanaru, C. Chapter 5: Systematic Reviews of Prevalence and Incidence. Available online: https: / / doi.org/10.46658/JBIMES-20-06 (accessed on 19 March 2021).

22. Maslach, C.; Jackson, S.E. Maslach Burnout Inventory. Research Edition; Consulting Psychologists Press: Palo Alto, CA, USA, 1981.

23. Moher, D.; Liberati, A.; Tetzlaff, J.; Altman, D.G.; The PRISMA Group. Preferred Reporting Items for Systematic Reviews and Meta-Analyses: The PRISMA Statement. PLoS Med. 2009, 6, e1000097. [CrossRef] [PubMed]

24. Out, J. Meanings of Workplace Bullying: Labeling Versus the Experiencing and the Belief in a Just World. A Dissertation Submitted to the Partial Fulfillment of the Requirements for the Degree of Doctor of Philosophy, University of Windsor, Windsor, ON, Canada, 2005.

25. Merecz, D.; Rymaszewska, J.; Mościcka, A.; Kiejna, A.; Jarosz-Nowak, J. Violence at the workplace-A questionnaire survey of nurses. Eur. Psychiatry 2006, 21, 442-450. [CrossRef] [PubMed]

26. Isaksson, U.; Graneheim, U.H.; Richter, J.; Eisemann, M.Å.S. Exposure to violence in relation to personality traits, coping abilities, and burnout among caregivers in nursing homes: A case-control study. Scand. J. Caring Sci. 2008, 22, 551-559. [CrossRef]

27. Merecz, D.; Drabek, M.; Moscicka, A. Aggression at the workplace-psychological consequences of abusive encounter with coworkers and clients. Int. J. Occup. Med. Environ. Health 2009, 22, 243. [CrossRef] [PubMed]

28. Spencer-Laschinger, H.K.; Grau, A.L.; Finegan, J.; Wilk, P. New graduate nurses' experiences of bullying and burnout in hospital settings. J. Adv. Nurs. 2010, 66, 2732-2742. [CrossRef]

29. Dikmetas, E.; Top, M.; Ergin, G. An examination of mobbing and burnout of residents. Turk. J. Psychiatry 2011, 22, 137-149.

30. Happell, B.; Koehn, S. Seclusion as a necessary intervention: The relationship between burnout, job satisfaction and therapeutic optimism and justification for the use of seclusion. J. Adv. Nurs. 2011, 67, 1222-1231. [CrossRef] [PubMed]

31. Murillo, J.C.; Orozco, V.; Ramos, M.; Parra, L. Prevalence of burnout syndrome and its relationship with workplace violence, in general practitioners of a hospital level I. Rev. Colomb. Salud Ocup. 2011, 1, 18-20.

32. Spence-Laschinger, H.K.; Finegan, J.; Wilk, P. Situational and dispositional influences on nurses' workplace wellbeing. Nurs. Res. 2011, 60, 124-131. [CrossRef]

33. Patrick, A.; Spence-Laschinger, H.K.; Wong, C.; Finegan, J. Developing and testing a new measure of staff nurse clinical leadership: The clinical leadership survey. J. Nurs. Manag. 2011, 19, 449-460. [CrossRef]

34. Sundin, L.; Hochwälder, J.; Lisspers, J. A longitudinal examination of generic and occupational specific job demands, and work-related social support associated with burnout among nurses in Sweden. Work 2011, 38, 389-400. [CrossRef]

35. Hensel, J.M.; Lunsky, Y.; Dewa, C.S. Exposure to client aggression and burnout among community staff who support adults with intellectual disabilities in Ontario, Canada. J. Intellect. Disabil. Res. 2012, 56, 910-915. [CrossRef]

36. Gascon, S.; Leiter, M.P.; Andrés, E.; Santed, M.A.; Pereira, J.P.; Cunha, M.J.; Albesa, A.; Montero-Marín, J.; García-Campayo, J.; Martínez-Jarreta, B. The role of aggressions suffered by healthcare workers as predictors of burnout. J. Clin. Nurs. 2012, 22, 3120-3129. [CrossRef]

37. Pranjić, N. Burnout and Predictors for Burnout among physicians in Bosnia and Herzegovina-survey and study. Acta Med. Acad. 2012, 35, 66-76.

38. Roldán, G.M.; Salazar, I.C.; Garrido, L.; Ramos, J.M. Violence at work and its relationship with burnout, depression and anxiety in healthcare professionals of the emergency services. Health 2012, 5, 193. [CrossRef]

39. Spence-Laschinger, H.K.; Wong, C.A.; Grau, A.L. The influence of authentic leadership on newly graduated nurses' experiences of workplace bullying, burnout and retention outcomes: A cross-sectional study. Int. J. Nurs. Stud. 2012, 49, 1266-1276. [CrossRef]

40. Galián-Muñoz, I.; Llor-Esteban, B.; Ruiz-Hernández, J.A. Violencia de los usuarios hacia el personal de enfermería en los servicios de urgencias hospitalarios. Factores de riesgo y consecuencias. Emergencias 2013, 26, 163-170.

41. Read, E.; Spence-Laschinger, H.K. Correlates of new graduate nurses' experiences of workplace mistreatment. JONA J. Nurs. Adm. 2013, 43, 221-228. [CrossRef] [PubMed]

42. Pineau-Stam, L.M.; Spence-Laschinger, H.K.; Regan, S.; Wong, C.A. The influence of personal and workplace resources on new graduate nurses' job satisfaction. J. Nurs. Manag. 2015, 23, 190-199. [CrossRef]

43. Threadgill, C.R. Perceptions of Workplace Bullying Among Practicing Registered Nurses; University of Southern Mississippi: Hattiesburg, MS, USA, 2014.

44. Hensel, J.M.; Lunsky, Y.; Dewa, C.S. Exposure to Aggressive Behaviour and Burnout in Direct Support Providers: The Role of Positive Work Factors. Available online: https:/ / www.sciencedirect.com/science/article/abs/pii/S0891422214004478 (accessed on 20 March 2021).

45. Hu, S.X.; Luk, A.L.; Smith, G.D. The effects of hazardous working conditions on burnout in Macau nurses. Int. J. Nurs. Sci. 2015, 2, 86-92. [CrossRef]

46. Trépanier, S.G.; Fernet, C.; Austin, S.A. Longitudinal investigation of workplace bullying, basic need satisfaction, and employee functioning. J. Occup. Health Psychol. 2015, 20, 105-116. [CrossRef] 
47. Hamdan, M.; Abu-Hamra, A. Workplace violence towards workers in the emergency departments of Palestinian hospitals: A cross-sectional study. Hum. Resour. Health 2015, 13, 28. [CrossRef]

48. Alameddine, M.; Mourad, Y.; Dimassi, H. A national study on nurses' exposure to occupational violence in Lebanon: Prevalence, consequences and associated factors. PLOS ONE 2015, 10, e0137105. [CrossRef] [PubMed]

49. Dal-Pai, D.; Lautert, L.; Cocaro de-Souza, S.B.; Palucci-Marziale, M.H.; Petri-Tavares, J. Violence, burnout and minor psychiatric disorders in hospital work. Rev. Esc. Enferm. USP 2015, 49, 457-464.

50. Menon, A.; Flannigan, C.; Tacchi, M.J.; Johnston, J. Burnout-or heartburn? A psychoanalytic view on staff Burnout in the context of service transformation in a crisis service in Leeds. Psychoanal. Psychother. 2015, 29, 330-342. [CrossRef]

51. Pintado-Cucarella, S.; Penagos-Corzo, J.C.; Casas-Arellano, M.A. Burnout syndrome in medical and obstetric perception of violence. Ginecol. Obstet. Mex. 2015, 83, 173-178.

52. Viotti, S.; Gilardi, S.; Guglielmetti, C.; Converso, D. Verbal aggression from care recipients as a risk factor among nursing staff: A study on burnout in the JD-R model perspective. BioMed Res. Int. 2015, 2015, 215267. [CrossRef]

53. Deniz, T.; Saygun, M.; Eroğlu, O.; Ülger, H.; Azapoğlu, B. Effect of exposure to violence on the development of burnoutsyndrome in ambulance staff. Turk. J. Med Sci. 2016, 46, 296-302. [CrossRef]

54. Galián-Muñoz, I.; Ruiz-Hernández, J.A.; Llor-Esteban, B.; López-García, C. User violence and nursing staff burnout: The modulating role of job satisfaction. J. Interpers. Violence 2016, 31, 302-315.

55. Llor-Esteban, B.; Sánchez-Muñoz, M.; Ruiz-Hernández, J.A.; Jiménez-Barbero, J.A. Violencia de los usuarios hacia los profesionales de salud mental y urgencias. Eur. J. Psychol. Appl. Leg. Context 2016, 9, 33-40. [CrossRef]

56. Anwar, M.M.; Elareed, H.R. Burnout among Egyptian nurses. J. Public Health 2017, 25, 693-697. [CrossRef]

57. Bawakid, K.; Abdulrashid, O.; Mandoura, N.; Shah, H.B.U.; Ibrahim, A.; Akkad, N.M.; Mufti, F. Burnout of physicians working in primary health care centers under Ministry of Health Jeddah, Saudi Arabia. Cureus 2017, 9, e1877. [CrossRef]

58. Karsavuran, S.; Kaya, S. The relationship between burnout and mobbing among hospital managers. Nurs. Ethics 2017, 24, 337-348. [CrossRef] [PubMed]

59. Hamdan, M. Burnout among workers in emergency Departments in Palestinian hospitals: Prevalence and associated factors. BMC Health Serv. Res. 2017, 17, 1-7. [CrossRef]

60. Portoghese, I.; Galletta, M.; Leiter, M.P.; Cocco, P.; D’Aloja, E.; Campagna, M. Fear of future violence at work and job burnout: A diary study on the role of psychological violence and job control. Burn. Res. 2017, 7, 36-46. [CrossRef]

61. Rafeea, F.; Al Ansari, A.; Abbas, E.M.; Elmusharaf, K.; Zeid, M.S.A. Violence toward health workers in Bahrain Defense Force Royal Medical Services' emergency department. Open Access Emerg. Med. OAEM 2017, 9, 113. [CrossRef] [PubMed]

62. Wongtongkam, N. An exploration of violence against paramedics, burnout and post-traumatic symptoms in two Australian ambulance services. Int. J. Emerg. Serv. 2017, 6, 134-146. [CrossRef]

63. Copeland, D.; Henry, M. The relationship between workplace violence, perceptions of safety, and professional quality of life among emergency department staff members in a level 1 trauma centre. Int. Emerg. Nurs. 2018, 39, 26-32. [CrossRef]

64. Coscun-Cenk, S. An analysis of the exposure to violence and burnout levels of ambulance staff. Turk. J. Emerg. Med. 2019, 19, 21-25. [CrossRef]

65. Kim, J.J.; Brookman-Frazee, L.; Gellatly, R.; Stadnick, N.; Barnett, M.L.; Lau, A.S. Predictors of burnout among community therapists in the sustainment phase of a system-driven implementation of multiple evidence-based practices in children's mental health. Prof. Psychol. Res. Pract. 2018, 49, 132. [CrossRef]

66. Looff, P.; Nijman, H.; Didden, R.; Embregts, P. Burnout symptoms in forensic psychiatric nurses and their associations with personality, emotional intelligence and client aggression: A cross-sectional study. J. Psychiatr. Ment. Health Nurs. 2018, 25, 506-516. [CrossRef]

67. Shier, M.L.; Graham, J.R.; Nicholas, D. Interpersonal interactions, workplace violence, and occupational health outcomes among social workers. J. Soc. Work 2018, 18, 525-547. [CrossRef]

68. Zaczyk, I.; Młocek, M.; Wilczek-Rużyczka, E.; Kwak, M. Patient aggression on the inpatient psychiatric wards and professional burnout among nurses Agresja chorych w zamkniętych oddziałach psychiatrycznych a wypalenie zawodowe pielegniarek. Pielegniarstwo Pol. 2018, 70. [CrossRef]

69. Aguilar-Nájera, O.; Zamora-Nava, L.E.; Grajales-Figueroa, G.; Valdovinos-Díaz, M.Á.; Téllez-Ávila, F.I. Prevalence of burnout syndrome in gastroenterologists and endoscopists: Results of a national survey in Mexico. Postgrad. Med. 2019, 132, $275-281$. [CrossRef] [PubMed]

70. Ajoudani, F.; Baghaei, R.; Lotfi, M. Moral distress and burnout in Iranian nurses: The mediating effect of workplace bullying. Nurs. Ethics 2019, 26, 1834-1847. [CrossRef] [PubMed]

71. Akram, Z.; Li, Y.; Akram, U. When Employees are Emotionally Exhausted Due to Abusive Supervision. A Conservation-ofResources Perspective. Int. J. Environ. Res. Public Health 2019, 16, 3300. [CrossRef] [PubMed]

72. Andela, M.; Truchot, D.; Huguenotte, V. Work environment and elderly abuse in nursing homes: The mediating role of burnout. J. Interpers. Violence 2019. [CrossRef]

73. El Ghaziri, M.; Dugan, A.G.; Zhang, Y.; Gore, R.; Castro, M.E. Sex and gender role differences in occupational exposures and work outcomes among registered nurses in correctional settings. Ann. Work Expo. Health 2019, 63, 568-582. [CrossRef] [PubMed]

74. Castro Negreiros, S.B. Asociación Entre Mobbing y Sindrome de Burnout en el Personal de Salud, Hospital Leoncio Prado Huamachuco2019; Universidad César Vallejo: Trujillo, Peru, 2019. 
75. Goussinsky, R.; Livne, Y. The role of coping resources in the relationship between mistreatment and job burnout: Evidence across two healthcare settings. J. Aggress. Maltreat. Trauma 2019, 28, 1250-1268. [CrossRef]

76. Jiménez, R.E.; Bachelet, V.C.; Gomolán, P.; Lefio, L.Á.; Goyenechea, M. Violence and burnout in health care emergency workers in Santiago, Chile: A survey-based cross-sectional study. Int. Emerg. Nurs. 2019, 47, 100792. [CrossRef] [PubMed]

77. De Looff, P.; Didden, R.; Embregts, P.; Nijman, H. Burnout symptoms in forensic mental health nurses: Results from a longitudinal study. Int. J. Ment. Health Nurs. 2019, 28, 306-317. [CrossRef]

78. Kim, W.; Bae, M.; Chang, S.J.; Yoon, J.H.; Jeong, D.Y.; Hyun, D.S.; Kim, C. Effect of burnout on post-traumatic stress disorder symptoms among firefighters in Korea: Data from the Firefighter Research on Enhancement of Safety and Health (FRESH). J. Prev. Med. Public Health 2019, 52, 345. [CrossRef] [PubMed]

79. Rayan, A.; Sisan, M.; Baker, O. Stress, workplace violence, and burnout in nurses working in King Abdullah Medical City during Al-Hajj season. J. Nurs. Res. 2019, 27, 26. [CrossRef]

80. Yasar-Hacer, T.; Ali, A. Burnout in physicians who are exposed to workplace violence. J. Forensic Leg. Med. 2020, 69, 101874. [CrossRef]

81. Vincent-Höper, S.; Stein, M.; Nienhaus, A.; Schablon, A. Workplace Aggression and Burnout in Nursing-The Moderating Role of Follow-Up Counseling. Int. J. Environ. Res. Public Health 2020, 17, 3152. [CrossRef]

82. INE. Escuela de Población Activa-Profesionales de la Salud [Internet]. 2018. Available online: https://www.ine.es/jaxiT3 /Datos.htm?t=4077 (accessed on 29 November 2020).

83. Tafjord, T. Managing Strong Emotions: Nurses' Recognition and Responses to Personal Emotions When Approaching Parents with Cancer and Their Dependent Children. Qual. Health Res. 2021, 1-16. [CrossRef]

84. Dillon, B.L. Workplace violence: Impact, causes, and prevention. Work 2012, 42, 15-20. [CrossRef] [PubMed]

85. Khadjehturian, R.E. Stopping the culture of workplace incivility in nursing. Clin. J. Oncol. Nurs. 2012, 16, 638-639. [CrossRef]

86. Frade-Mera, M.; Vinagre-Gaspar, R.; Zaragoza-García, I.; Viñas-Sánchez, S.; Antunez-Melero, E.; Álvarez-González, S.; MalpartidaMartín, P. Síndrome de burnout en distintas Unidades de Cuidados Intensivos. Enferm. Intensiv. 2009, 20, 131-140. [CrossRef]

87. Spencer-Laschinger, H.K.; Grau, A.L. The influence of personal dispositional factors and organizational resources on workplace violence, burnout, and health outcomes in new graduate nurses: A cross-sectional study. Int. J. Nurs. Stud. 2012, 49, $282-291$. [CrossRef] [PubMed]

88. West, C.P.; Dyrbye, L.N.; Erwin, P.J.; Shanafelt, T.D. Interventions to prevent and reduce physician burnout: A systematic review and meta-analysis. Lancet 2016, 388, 2272-2281. [CrossRef] 